\title{
A Similarity-Based Approach for the All-Time Demand Prediction of New Automotive Spare Parts
}

\author{
Daniel Steuer \\ Karlsruhe Institute of Technology \\ d.b.steuer@gmail.com \\ Peter Korevaar \\ IBM Germany \\ korevaar@de.ibm.com
}

\author{
Verena Hutterer \\ IBM Germany \\ verena.hutterer@de.ibm.com \\ Hansjörg Fromm \\ Karlsruhe Institute of Technology \\ hansjoerg.fromm@kit.edu
}

\begin{abstract}
The all-time demand prediction of new automotive spare parts is an area that has not received much attention in theory and practice in the past. This paper presents a new approach for tackling this important, but complex problem. The approach assumes that new parts will have similar demand patterns as comparable parts had in the past. For the study, the demand histories of 80.718 spare parts were provided from a German automotive manufacturer. In a first step, historical fullcycle demand patterns were clustered based on similarity, and a representative demand pattern (RDP) was determined for each cluster. In a second step, a classification model was trained on the historical data relating demand patterns with selected parts characteristics. In a third step, the classifier was used to predict for each new part to which cluster it belongs. The RDP of this cluster is then used to calculate the part's all-time demand. The developed models have been validated using standard quality measures used in analytics and will now be used in practice.
\end{abstract}

\section{Introduction}

According to Arthur D. Little [14], 23\% of the overall revenue and $54 \%$ of the profit of automotive manufacturers (OEMs) come from aftersales services and parts. On the other hand, 50-70\% of the spare parts inventory is often unused. This means that - even if the spare parts business is of so high importance forecasting and inventory planning methods are still inadequate and need to be improved.

The Automotive Industry has seen a steady increase in complexity over the last decades. The number of models and model variants that the OEMs release to market has grown significantly. And accordingly, the overall number of spare parts. Yet, the OEMs ensure the availability of spare parts for 15-20 years after end of production (EOP) of a vehicle.

With every new model, thousands of new parts are introduced for which the OEM has to predict the total demand of required spare parts over their complete lifecycles. This is an important prerequisite for sourcing decisions and price negotiations with the parts' suppliers.

Due to its complexity, lifecycle forecasting methods for spare parts have not received adequate attention in theory and practice. Academic research and industrial practice have largely concentrated on short-term methods for spare parts forecasting and inventory control, which are already rather sophisticated [3, 4, 23]. Lifecycle forecasting is still predominantly based on judgement.

Lifecycle forecasting for automotive spare parts bears some industry-specific problems, a mixture of market and technological dependencies. The major unknowns are:

- The market success (sales) of the primary product (the vehicle) itself

- The lifecycle (usage time) of the primary product and the time that the vehicle stays in the service responsibility of the OEM (before migrating to the independent aftermarket)

- The reliability and wear-out characteristics of parts (unknown especially for new parts), which determine the number of times a part gets replaced during the lifecycle of a vehicle

Each of these unknowns represents a challenge for itself. Altogether, they constitute a complex forecasting problem. As will be shown later, this brought us to choose an empirical approach in order to tackle this complex problem. 


\section{Related Work}

Related work is found in different areas of research: new product sales forecasting and end-of-life forecasting. All of these areas, however, cover only single aspects of our problem.

New product sales forecasting. This area has a long history in marketing research. Mas-Machuca et al. [15] give an overview of methods that have been applied for new product sales forecasting. They distinguish between (a) judgmental methods, (b) consumer and market research, (c) cause and effect models, and (d) artificial intelligence. An example of a combined judgmental /quantitative model is presented in Goodwin et al. [8] who estimate the parameters of a Bass model by regression with historical sales data of analogous products and assume that the parameters will be applicable for the new product. The Bass model [1] is a combination of exponential functions with two parameters, the coefficient of innovation and the coefficient of adoption, that allows to model a multitude of typical market penetration curves. The underlying assumptions of Bass make the model particularly suited for frequently purchased consumer products. Since the demand of spare parts is sporadic and extends over a long period of time, these models are of limited interest for our case.

Thomassey and Hapiette [21] describe a forecasting approach for new apparel items very similar to the approach we have chosen. The sales time series of historical items are clustered using self-organizing maps $(S O M)$. The clusters are representing prototypes for typical sales histories. A classification procedure based on probabilistic neural networks establishes a relation between the prototypes and individual item characteristics. For each new item (without any sales history), the classification procedure selects a prototype according to the new item's characteristics and applies the prototype's sales curve to predict the sales future of the new item. The approach of Thomassey and Hapiette was not limited by the number of predefined functions, but was applied in a fast-selling fashion environment. Therefore, a simple transferability to spare parts could not be assumed.

End-of-life forecasting. End-of-life forecasting differs from new product forecasting by the existence of a partial demand history [7, 22]. Jonas et al. [12] describe an approach that assumes that electronic spare parts of the same commodity exhibit similar demand patterns in the future, as they did in the past. They fit a connected line segment model (CLSM) consisting of three different phases to historical demand time series, normalize it, and obtain typical demand models (TDM) by clustering all normalized CLSMs. For demand prediction, they use a fuzzy similarity approach to fit the partial demand history of an already active part with the appropriate TDM.

Other approaches using partial demand histories are presented by Meixell and $\mathrm{Wu}$ [16] and by Bensing [2]. It is clear that for the prediction of new parts without demand history, end-of-life forecasting cannot be directly used, even if some of the applied techniques are very relevant.

\section{Our Approach}

Our approach is largely determined by the requirements that (a) no demand history is available for the new parts to be predicted, (b) no predefined demand shape shall be assumed for the prediction, and (c) no expert knowledge is needed in the process.

These requirements could be fulfilled in a three-step approach consisting of the steps clustering, classification, and prediction (Figure 1).

In step 1, a large amount of full-lifecycle demand patterns is first normalized and then clustered according to their shapes. A representative demand pattern (RDP) is selected to represent a cluster as the basis for the later prediction.

In step 2, a classification model is trained on the same historical data and relates certain characteristics of the parts with the identified clusters.

In step 3, the classification model is used to predict the appropriate cluster for a new part based on its characteristics. The representative demand pattern (RDP) of the predicted cluster is used to make a demand forecast for the new part.

\begin{tabular}{|c|c|c|}
\hline Clustering & Classification & $\begin{array}{l}\text { Assessment } \\
\text { \& Prediction }\end{array}$ \\
\hline $\begin{array}{l}\text { Find accurate cluster } \\
\text { within historical parts } \\
\text { - Select fitted demand } \\
\text { patterns from set of } 80 \mathrm{k} \\
\text { spare parts of OEM } \\
\text { - Shift demand to same } \\
\text { start } \\
\text { - Normalize demand } \\
\text { - Suitable distance } \\
\text { matrix } \\
\text { - Most promising } \\
\text { algorithm } \\
\text { - Optimal number luster } \\
\text { - Derive RDPs }\end{array}$ & $\begin{array}{l}\text { Classify parts via } \\
\text { inherent features } \\
\text { - Separate the data in } \\
\text { training and testing } \\
\text { data } \\
\text { - Select suitable } \\
\text { predictors } \\
\text { - Select suitable } \\
\text { classification algorithm }\end{array}$ & $\begin{array}{l}\text { Assess predicted } \\
\text { cluster \& derive forecast } \\
\text { - Assess predictor } \\
\text { quality } \\
\text { - Assess parts } \\
\text { allocation per cluster } \\
\text { - Predict all-time } \\
\text { demand by scaling the } \\
\text { RDP accordingly }\end{array}$ \\
\hline
\end{tabular}




\subsection{Data Source}

The data was already collected and its quality ensured before this research started. It consists of spare parts' master data, transaction data and replacement information. The monthly and yearly demand data is available from 1988 until today. The data sources were already prepared for modeling in general before this research started and a set of 80.718 records with at least 18 consecutive years of demand history within the given time period were initially identified as suitable parts from the prepared data set. Each of these records stands for one spare part, containing 243 different data fields and is uniquely identifiable by its key attribute, the spare part number. The other fields carry information regarding the historical demand and characteristics of each spare part.

Moreover, smoothed demand values of the individual parts were made available. Those demand values were created by a so called cubic spline fit of the historical aggregated yearly demand. Cubic spline fitting is a well-known method for non-linear regression modelling. A cubic spline is constructed of piecewise third-order polynomials $a_{i} x^{3}+b_{i} x^{2}+c_{i} x+d_{i}$ with appropriate coefficients $a_{i}, b_{i}, c_{i}$ and $d_{i}$ values $[6,9]$. Two of the four coefficients of each piecewise polynomial are used to link the pieces such that they fit seamlessly and are differentiable at the fitting points. The remaining coefficients are used to fit the full curve to the data such that a least square fit is made and the sum of the smoothed demand values equals the original demand values.

\subsection{Data Preparation}

Not every spare part in the given data set fulfills the criteria of a full demand lifecycle meaning that the start and the end of the lifecycle should be from 1988 until today. The earliest start of production (SOP) of one spare part was for example 1946 and the most recent one 2001, meaning that the parts are in different phases of their product lifecycle and we cannot see the whole lifecycle since demand data is only available for the years of 1988 until today, resulting in demand data that only represents a fraction of their lifecycle. Therefore, a selection of full-lifecycle parts out of all 80.718 spare parts is necessary. Since the model attempts to analyze and predict the all-time demand of spare parts, only parts with full demand lifecycles can be included for learning. To ensure full demand lifecycles the following criteria is implemented, resulting in 23.486 parts that can be included into the model:

$$
\text { - } \quad \mathrm{SOP}=1988+/-3 \text { years }
$$

- Number of historical demand years > 18 (together with the end of production (EOP) date, a long enough demand history is ensured) - $\quad$ EOP $<=2009$

In a second preparation step, the demand data of every part is shifted to the same start year in order to be able to compare the similarity of their demand lifecycle patterns. To correctly assess the similarity of demand patterns of different parts, the patterns need to have the same length and start at the same time otherwise the demand patterns are not comparable. Since the parts have different SOP and EOS (end of service) dates, an adjustment is necessary. To realize this adjustment, the demand or respectively the part's SOP date is shifted in time, such as if all parts would have the same SOP date.

The third step in the data preparation process for clustering is the normalization of the demand data. After shifting the demand data to the same starting point, the demand data $D_{i}$ of each part needs to be normalized in order to compare the demand patterns of the parts. The reason for normalization is to make sure that parts with the same shape of the demand pattern are clustered together even though some of them might have a high overall demand and some have a lower overall demand. Normalization is done by dividing the actual demand at the time $t$ by the sum of the demand of the full lifecycle for each individual part.

The last step within the data preparation for clustering is the creation of the distance matrix or dissimilarity matrix for the comparison of the similarity between each part in order to cluster them by similarity. Since the clustering is based on the similarity of demand patterns, the distance between every individual demand pattern to all other patterns needs to be calculated. In consideration of the fact that the spare part demand has already been shifted and normalized, the distance between two demand patterns is calculated by capturing the distance between the individual demand data points over time. Due to normalization, the total demand over time for each part equals 1 , which means for the measurement of distance of the individual parts that it can be basically described as the distance between histograms. When it comes to similarity or distance measurement of histograms, several distance measures exist. Within this research, the chi-square $(\chi 2)$ distance is used since it works well for the given scenario [18]. The $\chi 2$ histogram distance-measure originates from the $\chi 2$ test-statistic where it is used to test the fit between a distribution function and observed frequencies [20]. The $\chi 2$-distance, between two parts is represented by the following formula: 


$$
\chi^{2}=\frac{1}{2} \cdot \sum_{t=1}^{T} \frac{\left(x_{t}-y_{t}\right)^{2}}{\left(x_{t}+y_{t}\right)}
$$

where

$$
\begin{aligned}
& x_{t}=\text { normalized demand of part } x \text { at time } t \\
& y_{t}=\text { normalized demand of part } y \text { at time } t \\
& T=\text { total timespan of available demand data }
\end{aligned}
$$

The values of the $\chi 2$ - distance range between $[0,1]$, where a distance of 0 equals a high similarity and 1 equals low similarity. Thus, a quadratic distance matrix is created, which is the basis for the creation of the clusters.

\subsection{Clustering}

From the 80.718 available parts, 23.486 exhibited a full demand lifecycle and could therefore be included in the clustering process. A review of clustering techniques resulted in the selection of $k$-medoids clustering, since it is resistant to outliers and uses actual parts as centroids for the clusters. The available algorithms are PAM (partitioning around medoids) and CLARA (CLustering LARge Applications). We started with PAM and reserved CLARA for cases with run time capacity constraints.

As an iterative algorithm, PAM requires a set of parts that are handled as initial cluster centers [11]. For this purpose, we selected parts that have many similar parts and hence are likely to be good representative demand patterns (RDP). Based on the whole data set, a sub-set of parts with a high count of highly similar parts $\left(\chi^{2}\right.$-distance $\left.<0.01\right)$ is created and then a sample of $k$ (number of clusters) parts is randomly chosen as the initial cluster centers. The threshold for the parts belonging to the initial set is chosen to be the following:

$$
\#(\text { parts with } \chi 2-\text { distance }<0.01)>20 * k
$$

This means that only parts which have more than $k * 20$ parts, which have a $\chi^{2}$ - distance smaller than $\chi^{2}-$ distance $=0.01$ to the respective part, are eligible for the initial set. The threshold for $20 * k$ was set in order to keep the number of possibly eligible parts low and therefore to keep the computing time for PAM short.

The next step is the decision on the number of clusters $k$, which in PAM has to be made in advance. It is well known that "the choice of $\mathrm{k}$ is one of the most difficult problems of cluster analysis, for which no unique solution exists" [18]. For our case, we selected two quality measures, the Dunn index and the silhouette width, as decision support criteria.
One of the most frequently cited quality criterions for the identification of the optimal number of clusters is the Dunn Index [5]. The Dunn index aims at identifying clusters with high inter-cluster distance and low intra-cluster distance. The Dunn index for $\mathrm{K}$ clusters $C_{i}$ with $\mathrm{i}=1, \ldots, \mathrm{K}$ is defined by

$$
D U_{k}=\min _{i} \min _{j \neq i} f(i, j)
$$

where

$$
f(i, j)=\frac{d\left(C_{i}, C_{j}\right)}{\max _{m} \operatorname{diam}\left(C_{m}\right)}
$$

and $\mathrm{d}\left(\mathrm{C}_{\mathrm{i}}, \mathrm{C}_{\mathrm{j}}\right)$ is the dissimilarity/distance between cluster $C_{i}$ and $C_{j}$ and is defined by

$$
d\left(C_{i}, C_{j}\right)=\max _{\substack{x \in C_{i} \\ y \in C_{j}}} \operatorname{Distance}(x, y)
$$

and $\operatorname{diam}\left(\mathrm{C}_{\mathrm{m}}\right)$ is the intra-cluster function or diameter of the cluster defined by the equation

$$
\operatorname{diam}(C)=\max _{x, y \in C} \operatorname{Distance}(x, y)
$$

In short, the higher the values of the Dunn Index, the more compact and well separated are the clusters.

The silhouette width is based on a paper by Rousseeuw [19] which describes that clusters can be represented by their so-called silhouette $s(i)$, which is a comparison of its tightness and separation. This criterion signals which objects are positioned well within their cluster, and which ones are just somewhere in between clusters. To assess the relative quality of the clusters and to get an overview of how the data is configured, the entire clustering can be displayed by combining the silhouettes of all objects into a single plot. The average silhouette, called silhouette width, provides an evaluation of clustering validity, and might be used to select an 'appropriate' number of clusters.

The silhouette $s(i)$ itself is represented by the following formula

$$
s(i)=\frac{b(i)-a(i)}{\max \{a(i), b(i)\}}
$$

where $b(i)=$ avg. dissimilarity of $i$ and nearest neighbor cluster, and

$a(i)=$ avg. dissimilarity of $\mathrm{I}$ and the other objects in the cluster

The silhouette $s(i)$ can range from $[-1,1]$, where +1 and -1 mean that the object $i$ belongs to an adequate or 
inadequate cluster, respectively. If the silhouette value of the object $i$ belonging to the cluster A is close to zero, it means that the object $i$ can also be in the nearest neighbor cluster to A. The objective function, as like mentioned above, is the average of the silhouette $s(i)$ over the number of objects to be classified, and the best clustering is reached when this function is maximized [19].

We conducted experiments to find the optimal number $k$ of clusters according to the quality criteria. The first finding was that outlier parts existed which had rare demand patterns and distorted the shape of the individual clusters significantly. These outliers had to be removed before a smooth clustering could be conducted. All further investigations were now based on 15.621 parts without outliers.

Figure 2 shows the development of the silhouette width for an increasing number of clusters. We see a relatively flat optimum in the range of $25-29$ clusters.

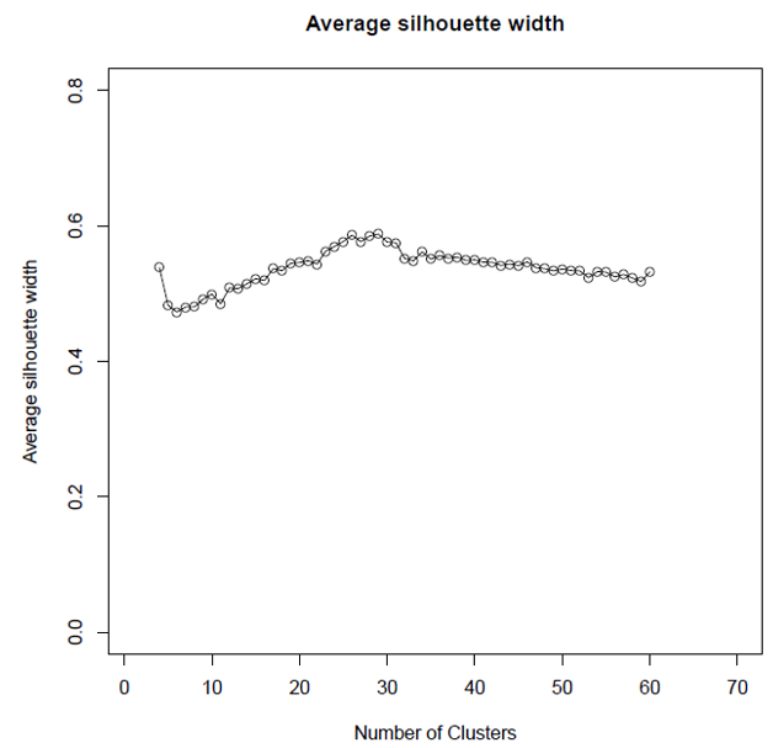

Figure 2: Silhouette width for different numbers of clusters $k$

The Dunn index showed an overall decline, but had some local optima in the range of $5-12$ (Figure 3 ).

We carefully analyzed all clusters for different $k$ in the range suggested by the silhouette width and the Dunn index. For this purpose, the shape of all demand lifecycle curves belonging to one cluster, together with the cluster centers and means, were visualized in graphs as shown in Figure 4. Particularly, we looked at the smoothness and meaningfulness of the clusters as they have been proposed by the clustering algorithm for different numbers $k$, and for the distribution of cluster sizes.

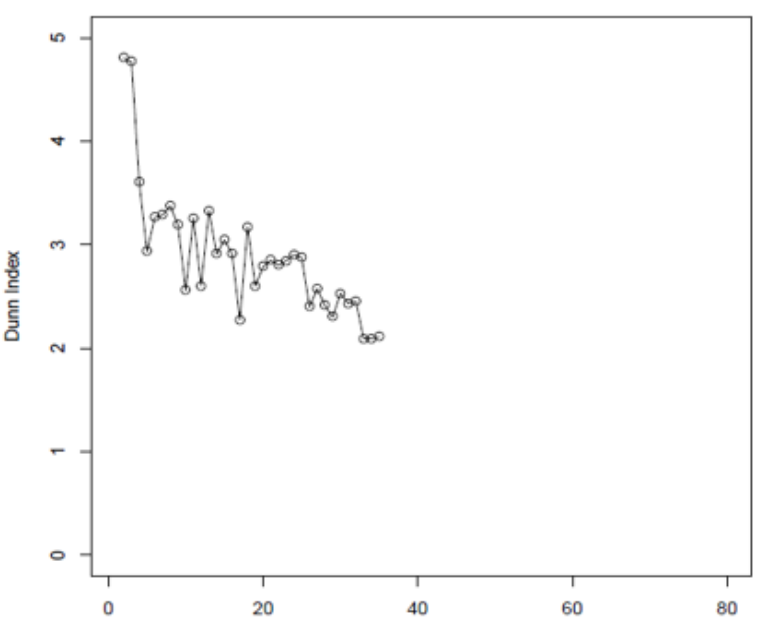

Figure 3: Dunn index for different numbers of clusters $\mathbf{k}$

A total number of $\mathrm{k}=8$ clusters, turned out to be the optimal number of clusters, since this number results in the highest cluster quality possible and at the same time represents as many distinctive demand patterns with a minimal number of repeating RDPs (Figure 5). The red curves are the cluster centers, and the green curves are the cluster means, which were used as the representative demand pattern (RDP) for that cluster.

Parts are fairly equally distributed across the 8 clusters. The proportion varies between ca. $8 \%$ and $18 \%$ of all parts per cluster. The largest clusters are cluster 2 with $15,75 \%$, cluster 3 with $17.92 \%$ and cluster 6 with $15,52 \%$ of all parts. Cluster 1 with $8,52 \%$ of all parts is the smallest cluster. The full parts distribution and the average $\chi^{2}$ - distance per cluster is shown in Figure 4.

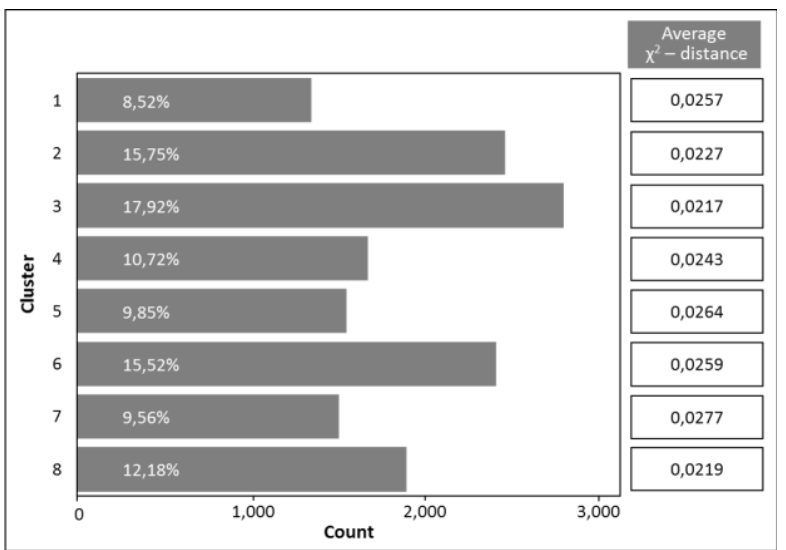

Figure 4: Absolute and relative cluster sizes (percentages of all parts) and average $x^{2}-$ distance for each cluster for the optimal number of $k=8$ clusters 


\subsection{Classification}

The objective of this classification is to associate a new spare part with one of the previously identified clusters, or representative demand patterns (RDP). As described above, there are 243 individual features per part which reflect parts and demand characteristics. In a first step, we identified the features that were most informative in the introduction phase of a new part. The resulting features were afterwards tested for their relevance by several experimental classifications and with the help of the IBM SPSS Modeler feature selection node. 7 features turned out to be significant. In a series of experiments, different classification algorithms were tested: Decision Tree (C 5.0),
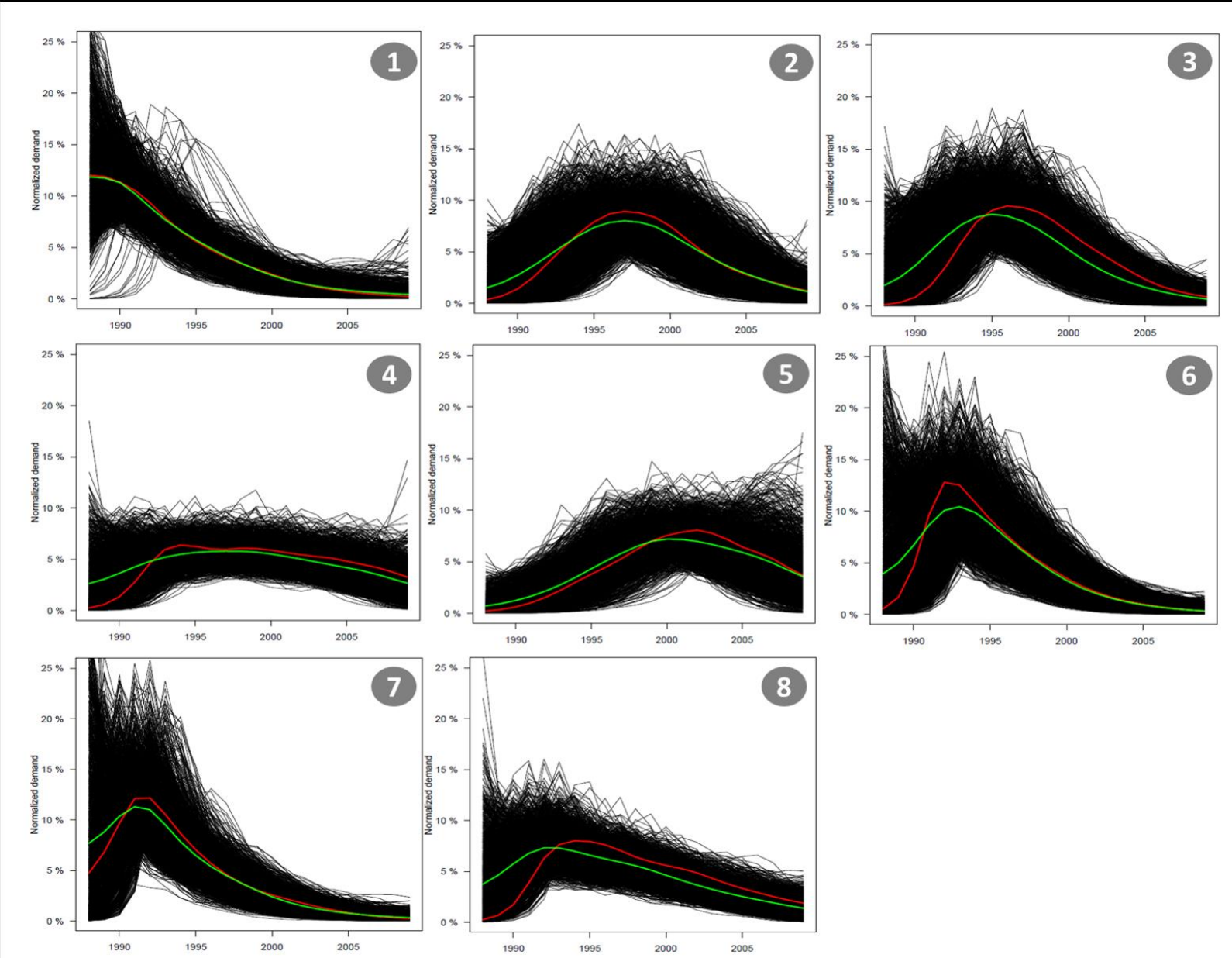

Figure 5. Optimal clustering output for $k=8$ including the cluster centers (darker) and cluster means (lighter). The latter are used as representative demand patterns (RDPs).

Artificial Neural Network(ANN), k-Nearest Neighbor $(\mathrm{kNN})$, Naïve Bayes, and Support Vector Machine (SVM). Each classifier was trained based on the 5-fold cross validation on stratified sample data. The Support Vector Machine (SVM) reaches the highest classification accuracy of $68.4 \%$, Naïve Bayes is second with a classification accuracy of $59.4 \%$ and the C 5.0 Decision Tree reaches an accuracy of 55.6\%, which ranks it third.
Figure 6 shows precision and recall for the best classifier SVM for each individual cluster and over all clusters. 


\begin{tabular}{|c|c|c|c|c|c|c|}
\hline K & False as $\mathrm{K}$ & True as $\mathrm{K}$ & $\mathrm{K}$ as False & Precision of $\mathrm{K}$ & Recall of $K$ & Accuracy \\
\hline 1 & 495 & 836 & $\begin{array}{r}322 \\
\end{array}$ & $62,81 \%$ & $72,19 \%$ & \\
\hline 2 & 692 & 1766 & 895 & $71,85 \%$ & $66,37 \%$ & \\
\hline 3 & 887 & 1912 & 983 & $68,31 \%$ & $66,04 \%$ & \\
\hline 4 & 534 & 1142 & 463 & $68,14 \%$ & $71,15 \%$ & \\
\hline 5 & 429 & 1109 & 391 & $72,11 \%$ & $73,93 \%$ & \\
\hline 6 & 695 & 1729 & 1014 & $71,33 \%$ & $63,03 \%$ & \\
\hline 7 & 519 & 974 & 321 & $65,24 \%$ & $75,21 \%$ & \\
\hline 8 & 680 & 1222 & 542 & $64,25 \%$ & $69,27 \%$ & \\
\hline & & & Total & $68,00 \%$ & $69,65 \%$ & $68,43 \%$ \\
\hline
\end{tabular}

Figure 6. Performance of SVM Classifier

\subsection{Demand Prediction}

After assessing how the parts can be clustered and which classification method creates the best prediction outcome, a model for the actual demand value needs to be implemented. The basis for this forecast are the normalized RDPs of each cluster that were identified during the clustering step. Since the RDPs are normalized demand patterns they must be scaled up in order to determine the real demand for the individual part. Scaling up the demand with the help of the RDPs requires at least the information about the first year of demand of the individual parts (named P1). As shown in the following formula, the all-time demand of a new spare part $n_{i}$ is calculated by accumulating the normalized demand of $n_{i}$ 's respective RDP up to year one and then divide the year one demand by this accumulated normalized demand value.

$$
\text { all-time demand }\left(n_{i}\right)=\frac{P 1\left(n_{i}\right)}{\int_{t=0}^{1} \operatorname{RDP}\left(n_{i}\right)}
$$

As the first year of demand is not known for new parts, it must be derived respectively. This can be done by using the planned number of produced vehicles in the first year, named $N V$, that contain $P l$, and the failure rate alpha of the new parts. Depending on the availability of data, the following alternatives for calculating $P 1$ are available:

1) Failure rate alpha is known, planned number of vehicles in first year $N V$ is known: $P 1=$ alpha* $N V / 2$.

2) Failure rate alpha is known, planned number of vehicles in first year $N V$ is unknown: Estimate $N V$ and use the formula under 1 ). If no such estimationis possible, $P l$ cannot be estimated(or, in other words: any estimation of $P 1$ would imply an estimation of $N V$ )

3) Failure rate alpha is unknown, planned number of vehicles in first year $N V$ is known: Estimate alpha from historical similar parts (same door, same

engine part, ...) and use the formulaunder 1). If no such estimation is possible, $P 1$ cannot be estimated (or, in other words: any estimation of $P 1$ would imply an estimation of alpha)

4) Failure rate alpha is unknown, planned number of vehicles in first year $P 1$ is unknown: Estimate $P 1$ from historical similar parts (same door, same engine part etc.). This is not an accurate method, since it assumes that $N V$ remains unchanged

In addition to the application for new parts, this model can also be very useful for parts with only a few years of historical demand. As mentioned in chapter 3 , in the early stages of a product life cycle, existing prediction models based on historical demand do not provide good forecasting results when it comes to all-time demand prediction. For these parts, the New Parts Model presented in this paper could provide a stable and trustworthy alternative.

\subsection{Implementation and Evaluation}

Simple tasks like the normalization of demand or the creation of the distance matrix were developed in PERL. The shifting of the demand to the same historic start year, the clustering of normalized and shifted demand patterns and the visualization of the clusters were developed using R. Feature selection, clustering, and classification was done using IBM SPSS Modeler.

As already mentioned, the evaluation of the algorithms was done step by step. The results were promising and in a range of accuracy that is sufficient for the demand planning of new parts. A full evaluation of the all-time demand prediction can only be made as more and more parts complete their lifecycles.

\section{Summary}

After an extensive literature study on long-term and new product planning, we decided to choose a similarity-based approach to tackle the problem of alltime prediction of new automotive spare parts.

We have found a few papers that have already used this specific approach [2, 12, 16, 21]. However, none of these are using their models for predicting new spare parts demand. Instead, they mostly rely on partial demand data to predict demand of parts in use. Further drawbacks of the research so far were the usage of partial demand patterns only for predicting similarity, the use of common distributionfunctions in order to strongly simplify the demand patterns, or the need of expert knowledge to create the final outcome of the prediction. 
Our chosen approach provides a fully qualified model for the prediction of new spare parts. It consists of three easily comprehensible steps: clustering, classification, and demand prediction. The first two steps could be evaluated and showed good results, so that the overall approach seems to be very well suited for the complex problem of all-time demand prediction of automotive spare parts. A generalization of the approach to other industrial situations seems possible.

\section{References}

[1] Bass, F. M. (1969). A New Product Growth Model for Consumer Durables. Management Science, 15, 215-227.

[2] Bensing, L. (2012). Life cycle prediction for spare parts at IBM spare parts operations. University of Twente.

[3] Boylan, J. E., \& Syntetos, A. A. (2008). Forecasting for Inventory Management of Service Parts. In Springer Series in Reliability Engineering (Vol. 8, pp. 479-506).

[4] Croston, J. D. (1972). Forecasting and Stock Control for Intermittent Demands. Journal of the Operational Research Society, 23(3), 289-303.

[5] Dunn, J. C. (1974). Well-Separated Clusters and Optimal Fuzzy Partitions. Journal of Cybernetics, 4(1), 95-104.

[6] Eilers, P. H. C., \& Marx, B. D. (1996). Flexible smoothing with B -splines and penalties. Statistical Science, 11(2), 89-121.

[7] Fortuin, L. (1980). The All-Time Requirement of Spare Parts for Service After Sales-Theoretical Analysis and Practical Results. International Journal of Operations \& Production Management, 1(1), 59-70.

[8] Goodwin, P., Dyussekeneva, K., \& Meeran, S. (2012). The use of analogies in forecasting the annual sales of new electronics products. IMA Journal of Management Mathematics, 24(4), 407-422.

[9] Hastie, T. J., \& Tibshirani, R. (1993). Varyingcoefficient Models. Journal of the Royal Statistical Society, 55(4), 757-796.

[10] Hong, J. S., Koo, H.-Y., Lee, C.-S., \& Ahn, J. (2008). Forecasting service parts demand for a discontinued product. IIE Transactions, 40(7), 640-649.
[11] Jain, A. K., Murty, M. N., \& Flynn, P. J. (1999). Data clustering: a review. ACM Computing Surveys, 31(3), 264 323.

[12] Jonas, T., Toth, Z. E., \& Dombi, J. (2015). A Knowledge Discovery Based Approach to Long-Term Forecasting of Demand for Electronic Spare Parts. Computational Intelligence and Informatics, 291-296.

[13] Kaufman, L. R., \& Rousseeuw, P. P. (1990). Finding groups in data: An introduction to cluster analysis. Hoboken NJ John Wiley \& Sons Inc. ISO 690.

[14] Little, A. D. (2008). Automotive After Sales 2015.

[15] Mas-Machuca, M., Sainz, M., \& Martinez-Costa, C. (2014). A review of forecasting models for new products. Intangible Capital, 10(1), 1-25.

[16] Meixell, M., \& Wu, S. (2001). Scenario analysis of demand in a technology market using leading indicators. Semiconductor Manufacturing, IEEE, 14(1), 65-75.

[17] Moore, J. R. (1971). Forecasting and Scheduling for Past-Model Replacement Parts. Management Science, 18(4Part-I), B-200-B-213.

[18] Pele, O., \& Werman, M. (2010). The quadratic-chi histogram distance family. In Lecture Notes in Computer Science (Vol. 6312 LNCS, pp. 749-762). School of Computer Science. The Hebrew University of Jerusalem.

[19] Rousseeuw, P. J. (1987). Silhouettes: A graphical aid to the interpretation and validation of cluster analysis. Journal of Computational and Applied Mathematics, 20(C), 53-65.

[20] Snedecor, G. W. \& Cochran, W. G. (1977). Statistical methods applied to experiments in agriculture and biology. Citation Classics, 19, 1.

[21] Thomassey, S., \& Happiette, M. (2007). A neural clustering and classification system for sales forecasting of new apparel items. Applied Soft Computing Journal, 7(4), $1177-1187$.

[22] Teunter, R. H., \& Fortuin, L. (1999). End-of-life service. International Journal of Production Economics, 59(1), 487-497.

[23] Zhu, S., Dekker, R., Van Jaarsveld, W., Renjie, R. W., \& Koning, A. J. (2017). An improved method for forecasting spare parts demand using extreme value theory. European Journal of Operational Research, 261(1), 169-181. 